\title{
Are We Prescribing the Right Diets and Drugs for CAD, T2D, Cancer and Obesity?
}

Fleming RM ${ }^{1 *}$, Fleming $\mathrm{MR}^{1}$ and Chaudhuri $\mathrm{TK}^{2}$

${ }_{1}^{1}$ FHHI-OmnificImaging-Camelot, USA

2Eastern Virginia Medical School, Virginia

*Corresponding author: Richard M Fleming, PhD, MD, JD, Los Angeles, CA, USA, Tel:

818-210-6930; Email: DrRichardMFleming@gmail.com

\section{Letter to Editor}

Volume 2 Issue 2

Received Date: August 21, 2019

Published Date: August 27, 2019

DOI: $10.23880 /$ ijnmrs-16000115
Keywords: FMTVDM; CAD; T2D; Cancer; Obesity

Abbreviations: CAD: Coronary Artery Disease; T2D: Type 2 diabetes; CRP: C-Reactive Protein; MACE: Major Adverse Cardiac Events.

\section{Letter to Editor}

One cannot read a medical journal [1] or participate in social media without becoming increasingly aware of the dichotomy that has taken place in the debate over what constitutes a healthy and non-healthy diet. Despite what is undoubtedly a strong concern by many over how to best address the ever-increasing incidence of "inflammatory" diseases including coronary artery disease (CAD), adult onset diabetes mellitus (T2D), obesity and certain types of cancer, there remains considerable disagreement.

The clinical treatment of these diseases for hundreds of thousands to millions of people around the world depends upon when and where the patient's physician was trained. For many the focus remains on measuring cholesterol levels and other blood tests looking for inflammation. Many of the currently published dietary studies emphasize looking for improvement in cholesterol or inflammation levels to support the success of their dietary regimens or drug treatment. It is clear that many clinicians depend upon these blood tests to determine if their treatment (diet, medication, surgery, etc.) for obesity, T2D, Cancer and/or CAD is successful.

These blood tests, which are commonly checked in the "fasting" state focus on looking for reductions in specific cholesterol levels, glucose, hemoglobin A1C (glycosylated hemoglobin), fibrinogen, homocysteine, c-reactive protein (CRP) and a series of other tests. The relationship of these and numerous other factors and their role of CAD are shown in Figure 1 and are discussed in detail elsewhere [2-4].

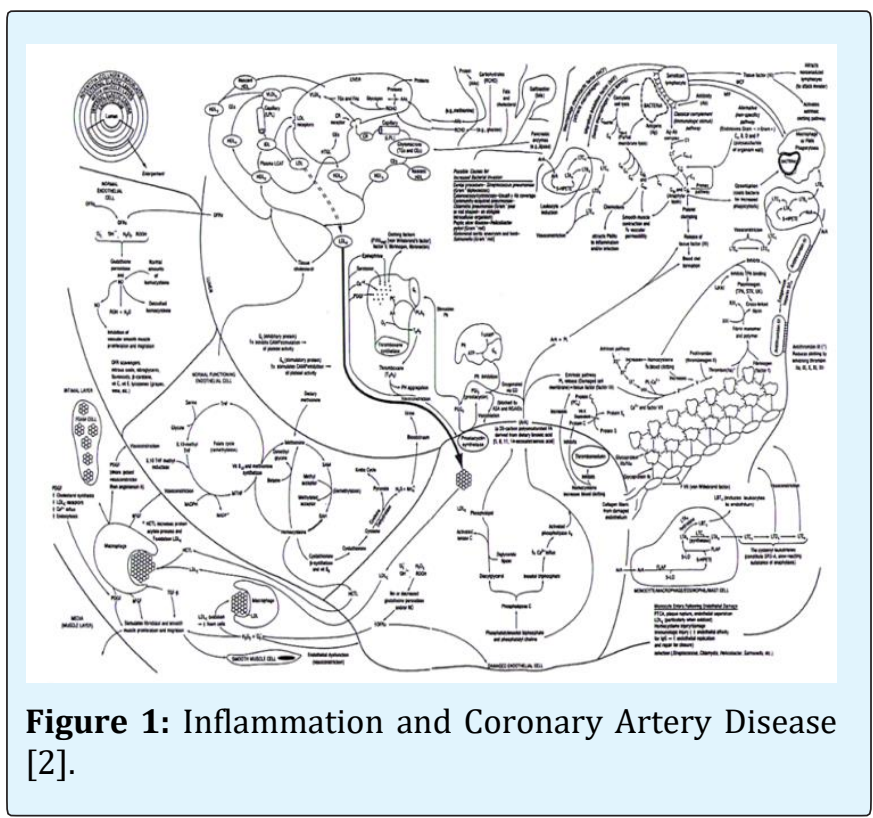

The measurement of these blood tests demonstrate an expansion in thinking from the simple, cholesterol-is-thesole-cause-of-heart-disease, to cholesterol along with a host of other factors producing an inflammatory process [2], all of which are ultimately responsible to varying degrees for the impaired blood flow through the coronary arteries in any given individual. The subsequent build-up 
of inflammatory plaques ultimately impairs the ability of the coronary arteries to relax to carry more blood flow to the heart, which in turns compromises the heart when the metabolic demands of the heart increase [3] producing major adverse cardiac events (MACE).

For many clinicians responsible for the care and treatment of patients, this is unfortunately the event horizon. What remains up for debate is how the various diets and medications selected for treatment of patients actually impact CAD, T2D, obesity and at least certain types of cancer. Should patients be prescribed a diet consistent with the American Heart Association recommendations? Should they be prescribed vegan or vegetarian diets? Should they be prescribed KETO genic diets? Should they be prescribed statins and PCSK9 inhibitors? Are we prescribing the right diets? Are we over prescribing medications? Are we using the right tests to know?

As established (Figure 2) in 2008 [4], measuring changes in cholesterol and inflammation do not tell us what is actually happening inside the walls of any given patients coronary arteries. The disconnect between blood tests and actual CAD raises serious questions about using these blood tests to determine the effect of diets or medications for CAD, T2D, cancer or obesity.

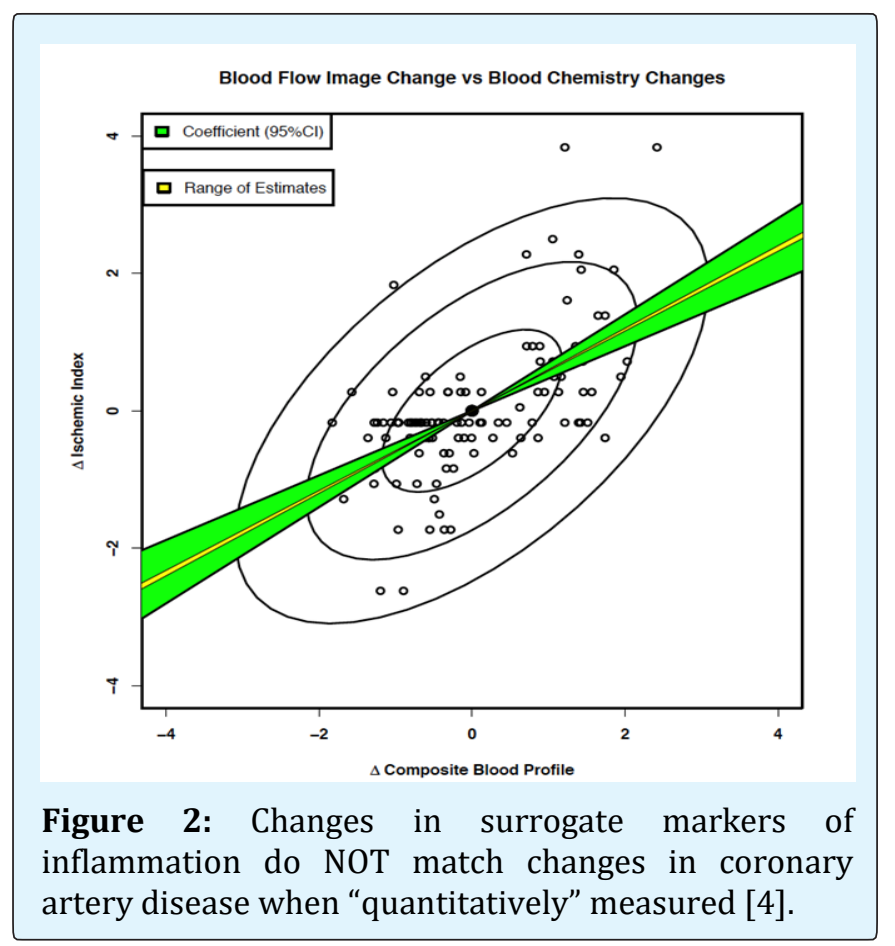

Quantitative measurement of changes in CAD and cancer requires a method capable of accurately, consistently and reproducibly measuring these changes [5]. Similarly, we can also measure the extent to which treatment of T2D and obesity is associated with changes in cancer and CAD. As we attempt to expand our knowledge and understanding into the causes and treatment of CAD, T2D, Cancer and Obesity, it becomes increasingly important that we measure the actual outcome of these treatments. If we are to truly "do no harm", we must measure the effect of our treatments to provide the best medical care possible for our patients, making it possible to prescribe the best diet and lifestyle choices possible, while at the same time judiciously limiting the use of medications to those instances where they are truly beneficial and only for the amount of time they prove to be valuable.

Conflicts of Interest: FMTVDM was issued to the primary author. All figures reproduced with expressed consent of primary author.

\section{References}

1. GBD 2017 Diet Collaborators (2019) Health effects of dietary risks in 195 countries, 1990-2017: a systematic analysis for the Global Burden of Disease Study. Lancet 393(10184): 1958-1972.

2. Fleming RM (2000) The Pathogenesis of Vascular Disease. Chapter 64, In: Chang JB (Eds.), Textbook of Angiology, Springer, New York, pp: 787-798.

3. Fleming RM (2000) Atherosclerosis: Understanding the relationship between coronary artery disease and stenosis flow reserve. Chapter 29, In: Chang JB (Eds.), Textbook of Angiology, Springer-Verlag, New York, NY, pp: 381-387.

4. Fleming RM, Harrington GM (2008) What is the Relationship between Myocardial Perfusion Imaging and Coronary Artery Disease Risk Factors and Markers of Inflammation? Angiology 59(1):16-25.

5. (2017) The Fleming Method for Tissue and Vascular Differentiation and Metabolism (FMTVDM) using same state single or sequential quantification comparisons. Patent Number 9566037. 